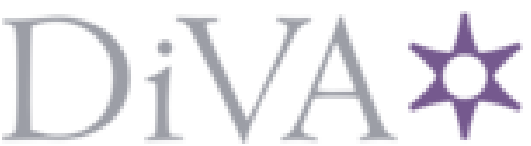

http://www.diva-portal.org

Postprint

This is the accepted version of a paper presented at IEEE/RSJ International Conference on Intelligent Robots and Systems (IROS).

Citation for the original published paper:

Chernik, C., Tajvar, P., Tumova, J. (2021)

Robust Feedback Motion Primitives for Exploration of Unknown Terrains

In:

N.B. When citing this work, cite the original published paper.

Permanent link to this version:

http://urn.kb.se/resolve?urn=urn:nbn:se:kth:diva-304336 


\title{
Robust Feedback Motion Primitives for Exploration of Unknown Terrains
}

\author{
Charles Chernik $^{1 *}$, Pouria Tajvar ${ }^{1 *}$, Jana Tumova $^{1}$
}

\begin{abstract}
Unknown properties of a robot's environment are one of the sources of uncertainty in autonomous navigation. This uncertainty has to be accounted for when modelling robot dynamics. For ground vehicles in particular, terrain structure is one of the main environmental factors that can strongly influence the dynamics. Therefore, to ensure the ability of a robot to safely and efficiently navigate new environments, robust motion planning and control systems are needed. This paper investigates a data-driven approach to planning and control based on construction of robust motion primitives (MPs) and corresponding feedback rules that ensure a bounded error along the planned trajectory. The approach is tested in an exploration scenario in which a robot systematically inspects an area consisting of several terrain types with the aim of recognizing changes in dynamical properties, learning new dynamics models when such changes are detected and recording that information for future use. The advantage of incorporating the collected data into motion planning in multi-terrain environments is illustrated via simulation.
\end{abstract}

\section{INTRODUCTION}

Navigation in diverse outdoor environments with various topological, environmental, or even gravitational conditions is currently among the high-priority technological challenges, for instance, for NASA and the global space sector in general [1]. As most of space exploration is still performed by ground mobile robots (e.g., Mars Exploration Rovers) without immediate human supervision and the ambition of humanity constantly grows, reliable autonomy continues to be a matter of key importance.

One of the main challenges in autonomous navigation is to make sure that a planned trajectory is feasible given the robot's dynamical limitations. Finding such a feasible trajectory requires a model of robot dynamics that is highly uncertain, largely due to the fact that the robot may encounter and have to navigate through various previously unseen kinds of terrain, which is often the case in planetary exploration context. Furthermore, Unmanned Ground Vehicles (UGVs) are usually subject to nonholonomic constraints. Consequently, to account for both the changing conditions and the robot's own limitations, data-driven methods of dynamics modelling are needed, as well as planning algorithms that will incorporate the learned models to obtain efficient and robust motion plans with safety guarantees.

In this work, we propose a data-driven approach to integrated motion planning and control in an unknown area

\footnotetext{
${ }^{1}$ Charles Chernik, Pouria Tajvar, and Jana Tumova are with the Division of Robotics, Perception and Learning, School of Electrical Engineering and Computer Science, KTH Royal Institute of Technology, Stockholm, Sweden. The authors are also affiliated with Digital Futures. \{chernik, tajvar, tumova\}ekth.se

${ }^{*}$ Equal contribution
}

exploration scenario. Similarly as in our previous work [2], the approach consists of constructing a library of robust motion primitives (MPs) by sampling from the robot dynamics and fitting a piece-wise linear model. Using these primitives, we find feasible and cost-effective motion plans, and then derive feedback rules that keep the deviation from the planned trajectory bounded in the presence of dynamics uncertainty. In contrast to the previous work, the goal in this scenario is to identify the dynamics in regions of the area with different terrains.

\section{A. Related Work}

Various approaches to the problem of motion planning for nonholonomic vehicles have been proposed in recent years. The approaches range from those relying on simplified models of vehicle dynamics, e.g., the bicycle model [3], to feedback-based methods that use MPs constructed from the real vehicle dynamics [4], [5]. A subset of works focuses specifically on the problem of navigating uncertain or unknown environments [6]-[8].

The nonholonomic nature of UGVs makes the control design for them nontrivial in the presence of disturbance and uncertainty. Therefore, it is also necessary that a robust control algorithm is employed, allowing to keep the position uncertainty bounded as the vehicle moves along the planned trajectory. A lot of recent research on control design has been dedicated to model-based approaches [9]-[11]. While motion planning and control are often regarded as separate problems, with most works focusing on either one or the other, some combined approaches have also been proposed [2], [12]-[14].

A review of earlier works on terrain classification is carried out in [15]. These works have mainly investigated methods to associate the real-time measurements with one class from a pre-defined set of terrains, e.g., asphalt, sand, or gravel [16], [17]. Alternatively, methods have been proposed to rate the terrains' traversability based on visually distinctive features such as steepness, roughness and discontinuity [18]. The reactive nature of such approaches allowed for adaptation of the control policy for each terrain, however, it did not enable incorporating the terrain properties into path planning. As a result, the problem of terrain aware path optimization remained a challenge; this problem is being studied for ground vehicles, mainly through visual assessment of the terrains [19], [20]. We are now proposing an alternative to visual assessment by trial traversing to learn the vehicle dynamics on different terrain types and constructing feasible MPs for each terrain type that can be used for motion 
planning. This can be particularly beneficial for identifying the dynamics on terrains where prior experience does not exist.

\section{B. Contribution}

The main contribution of this work is an exploration approach for a multi-terrain environment which involves construction of MPs specific to the terrains where the UGV dynamics is not known a-priori. The advantage of terrainspecific MPs in comparison to adaptive/robust control is that the choice of MPs can be tailored during the planning phase for cost optimization. For example, the differences in the grip between different terrains can affect various aspects of feasible maneuvers such as the turning radius and velocity limits; similarly, traversing energy consumption can be significantly different between terrains such as rock vs sand. The MPs are constructed with feedback controllers that ensure bounded reachable sets that, in turn, allow for long term planning with bounded deviation, and hence robust and safe control. We test the described approach on Erle Rover UGVs on different terrains, demonstrating its benefits.

\section{Preliminaries}

Let us denote the set of real numbers by $\mathbb{R}$ and an $n$ dimensional vector space by $\mathbb{R}^{n}$. The Minkowski sum $A \oplus B$ of two sets $A \subset \mathbb{R}^{n}$ and $B \subset \mathbb{R}^{n}$ refers to the following set:

$$
A \oplus B=\{a+b \mid a \in A, b \in B\} .
$$

A zonotope $Z(\mu, G) \subset \mathbb{R}^{n}$, where $\mu \in \mathbb{R}^{n}$ and $G \in$ $\mathbb{R}^{m \times n}$, refers to the centrally symmetric set

$$
Z(\mu, G)=\left\{x \in \mathcal{X} \mid x=\mu+G \omega, \omega \in[-1,1]^{n}\right\},
$$

where $[-1,1]^{n} \subset \mathbb{R}^{n}$ represents the Cartesian product of closed $[-1,1]$ intervals. We refer to $\mu$ as the center of the zonotope and $G$ as the set of generators. Z. $\mu$ and Z.G are used to refer to the corresponding parameters of the zonotope $Z$. We use the following zonotope operations:

$$
\begin{array}{ll}
A \times Z, \text { where } & (A \times Z) \cdot \mu=A Z \cdot \mu, \\
& (A \times Z) \cdot G=A Z \cdot G ; \\
Z \oplus Z^{\prime}, \text { where } \quad\left(Z \oplus Z^{\prime}\right) \cdot \mu=Z \cdot \mu+Z^{\prime} \cdot \mu, \\
& \left(Z \oplus Z^{\prime}\right) \cdot G=\left[Z \cdot G \mid Z^{\prime} \cdot G\right],
\end{array}
$$

where $\left[Z . G \mid Z^{\prime} . G\right]$ denotes the concatenation of the two matrices along their columns.

\section{Problem Statement}

Consider a UGV in an environment $\mathcal{E} \subset \mathbb{R}^{2}$ that is divided into $n_{r}$ regions $\mathcal{R}_{i}$ corresponding to different types of terrain, and an obstacle region $\mathcal{O} \subseteq \mathcal{E}$, such that $\mathcal{E}=\bigcup_{i=1}^{n_{r}} \mathcal{R}_{i} \cup \mathcal{O}$. Suppose that the boundaries between the regions and between the regions and the obstacles are obtained, e.g., through prior aerial inspection or satellite imaging (see example in Fig. 1). We thus assume that given $p \in \mathcal{E}$, the region that $p$ belongs to is known. However, the dynamics of the UGV in the region is not known and it is

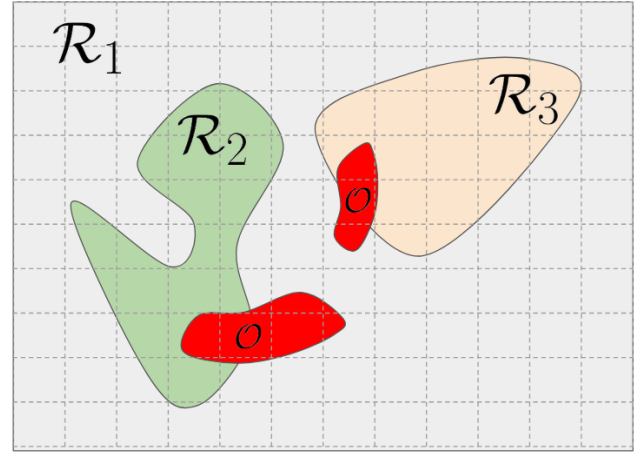

Fig. 1: An environment with two obstacles and three regions. Gridding of the environment is for exploration strategy planning and provides cells where dynamics identification will be carried out.

different on different types of terrain. In region $\mathcal{R}_{i}$, it can be expressed as:

$$
\dot{x}=f_{i}(x, u)+w,
$$

where $x=\left(p_{x}, p_{y}, z\right)^{T}$ is the state of the UGV that includes its position in the environment $p=\left(p_{x}, p_{y}\right) \in \mathcal{R}_{i} \subset E$ and its internal state $z \in \mathbb{R}^{n_{z}}$, e.g., velocity and orientation. $u \in$ $\mathcal{U} \subseteq \mathbb{R}^{n_{u}}$ is the system actuation input and $w \in \mathcal{W} \subseteq \mathbb{R}^{n_{w}}$ is a bounded disturbance. Functions $f_{i}$ cannot be accessed explicitly, but the current state $x$ can be measured.

The mission of the UGV is to identify a set of MPs for $f_{i}$ on each type of terrain $\mathcal{R}_{i}$ while avoiding collision with the obstacles, and use the identified MPs in planning for any subsequent mission, allowing for efficient plans that avoid costly terrains in terms of, e.g., traversing energy or time. One of the main challenges during the exploration and identification phase is that obstacle avoidance needs to be guaranteed despite the unknown dynamics.

\section{APPROACH OVERVIEW}

This section gives an overview of the approach to the problem defined above (see Fig. 2). We begin from a known initial state and a grid representation of the entire area. The grid is defined to be fine enough that for every terrain region, there are some grid cells that are fully enclosed in that region, as in Fig. 1. Since the boundaries of all regions are known, the smallest region can be easily identified and the appropriate size of the grid cells subsequently determined.

With this representation we can use a discrete planner to find a plan that visits at least one grid cell in every region. We refer to this discrete plan as the exploration strategy and assume it as given. In addition, we assume to be given a default dynamics model of the UGV, which is a conservative estimate that assumes large uncertainty and thus guarantees avoidance of obstacles. Such model can also be derived in the UGV's initial state as we explain in Section V-D.

Following the exploration strategy, we plan a path to the next target grid cell. Such a plan is implemented as a sequence of MPs derived as detailed in Section V-C, either for the default dynamics or for the terrain-specific dynamics 


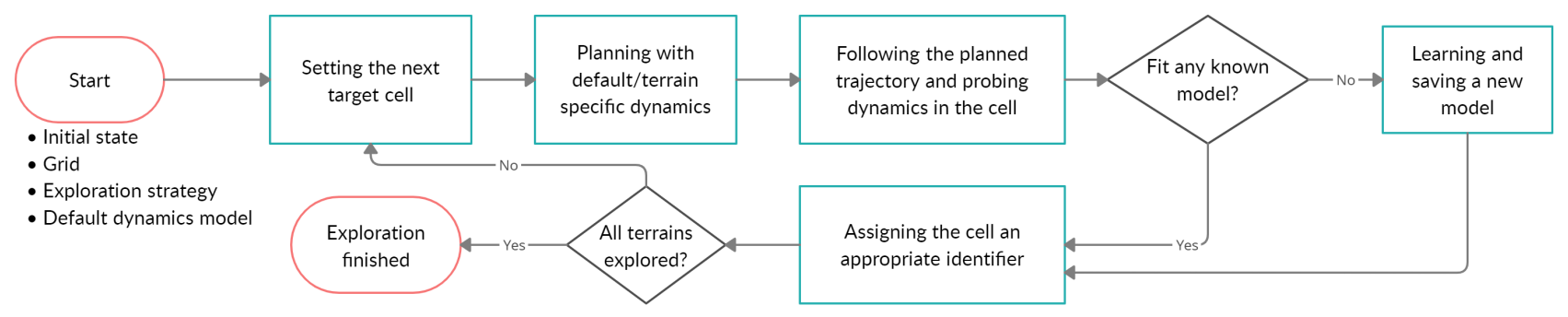

Fig. 2: Approach overview.

when available. This plan can be verified for being robustly collision free via computation of the reachable sets for each MP (see Section V-B).

After arriving in the target grid cell, the local dynamics is probed as explained in Section V-A. If the local dynamics matches any of the existing models, the model is assigned to the corresponding terrain type, otherwise a new model is constructed for the terrain. The exploration is concluded when a model is assigned to every terrain type.

\section{Solution}

\section{A. Modelling the dynamics}

While the underlying dynamics of the $\operatorname{UGV} f_{i}(s, u)$ is nonlinear in $\mathcal{R}_{i}$, we attempt to construct a set of locally linear models to approximate a discretization of the dynamics. Our motivation comes from the fact that for a linear observable system, the system state $x$ can be recovered from a sequence of measurements. In addition, the model of such a system can be identified by applying a persistently exciting input to the system [21].

Let us define $F(s, u)$ as the zero-order-hold $(\mathrm{ZOH})$ time discretization of the system (3) with duration $\tau$ as follows:

$$
\begin{aligned}
F(x, u)=\{\mathbf{x}(\tau) \mid & \mathbf{x}(0)=x, \\
& \forall 0 \leq t \leq \tau, \mathbf{u}(t)=u, \mathbf{w}(t) \in \mathcal{W} \\
& \dot{x}(t)=f(\mathbf{x}(t), \mathbf{u}(t))+\mathbf{w}(t)\}
\end{aligned}
$$

i.e. $F(x, u)$ is the set of all of the states that may be reached from state $x$ while applying the input $u$ for time $\tau$.

To construct local approximations of $F$, we decompose the input space $\mathcal{U}$ of the system into a set of cells $U_{j}$, $j \in\{1, \ldots, M\}$. For each $U_{j}$, we collect a set of data samples by exerting inputs $u \in U_{j}$ for the fixed duration $\tau$, where each sample becomes the triple $(x, u, F(x, u))$. To ensure that exerting an input is safe, i.e. collision free and not leading out of the desired region, we rely on the Lipschitz continuity assumption: $\left|F\left(x, u_{2}\right)-F\left(x, u_{1}\right)\right|<L\left|u_{2}-u_{1}\right|$. And since we initially know that for a stationary UGV we have $F(x, \mathbf{0})=x$, we know the initial set of safe inputs to sample from. After obtaining a rough initial model, we will instead use reachable set analysis, explained in section V-B, to verify the safety of an input. We refer to the process of safe sample collection in a region as probing.

Approximating $F$ in a position $p \in \mathcal{R}_{i}$ for the inputs $u \in$ $U_{j}$, using the set of collected samples $D$, can be formulated as a linear programming (LP) problem:

$$
\begin{array}{rl}
\min _{A, B, c, w} & w \\
\text { s.t. } & \forall(x, u, F(x, u)) \in D, \\
& \|F(x, u)-A x+B u+c\|_{\infty} \leq w .
\end{array}
$$

As a result, the UGV dynamics is expressed in the form:

$$
\bar{F}_{i, j}(x, u)=\{A x+B u+c\} \oplus \mathbf{W},
$$

where $\mathbf{W}$ is a hyper-rectangle that corresponds to the linearization error bounds, and $\forall p \in \mathcal{R}_{i}, u \in U^{j}, F(x, u) \subseteq$ $\bar{F}_{i, j}(x, u) . \mathbf{W}$ can be calculated again by assuming Lipschitz continuity as detailed in [2]. Intuitively, as the number of samples in $D$ grows, the size of $\mathbf{W}$ approaches the result of the optimization problem (5): $w$.

\section{B. Reachable set computation}

To be able to ensure safety during the execution of an input, we should verify that all of the states that can be visited by the UGV are indeed within the safe area $\mathcal{E} \backslash \mathcal{O}$. This can be done through computation of the reachable set for the obtained locally linear dynamics. Furthermore, we will use the computed reachable set to design a feedback controller for the MPs to ensure bounded uncertainty over arbitrarily long trajectories.

We use zonotopes to represent the reachable sets as they are closed under linear transformations; i.e. starting from a zonotope set $Z_{x}$ in the state space and having a zonotope input set $Z_{u}$, the reachable set $Z_{\text {reach }}$ of the linear system $\bar{F}_{i, j}(x, u)$ is also a zonotope and can be computed as:

$$
Z_{\text {reach }}=A \times Z_{x} \oplus B \times Z_{u}+c \oplus \mathbf{W} .
$$

We note that using zonotopes is also very computationally efficient given that the Minkowski sum for zonotopes is simply obtained by concatenating the columns of the two generator matrices. In practice our approach takes less than $1 \mathrm{~ms}$ to compute the reachable set for each motion primitive.

\section{Constructing feedback motion primitives}

We construct an MP for each input cell $U_{j}$ with a feedforward part being the central value of the $U_{j}$ and a feedback part that is computed after the locally linear model for $U_{j}$ is computed. We know that for systems that are not asymptotically stable, including the UGV, the reachable set tends to be larger than the starting set; as a result, as the 
trajectories get longer, potentially the deviation from the prescribed trajectory also grows. Therefore, we are motivated to design a feedback term that prevents this. As seen in Eq. (7), the reachable set is composed of two main components, namely the set resulting from the state evolution $A \times Z_{x}$ and the set from the input application $B \times Z_{u}$. A linear feedback controller $u=-\kappa x$ can be used to potentially shrink the reachable set size by counteracting the state deviation from the center of the state zonotope $Z_{x}$; the reachable set of the closed loop system becomes:

$$
Z_{\text {reach }}^{c l}=(A-B \kappa) \times Z_{x}+B \kappa Z_{x} \cdot \mu+B Z_{u} \cdot \mu+c \oplus \mathbf{W} .
$$

However, UGVs are in most cases under-actuated systems, and, as a result, $B \times Z_{u}$ is a degenerate set and such a controller $u=-\kappa x$ cannot be found to pose an arbitrary bound on the size of the reachable set in one time step. Let us, therefore, introduce the $k$-step extension of $\bar{F}_{i, j}(x, u)$. We define $F_{i, j}^{1}=F_{i, j}$ and

$$
F_{i, j}^{k+1}\left(x,\left[u_{1}^{\top} \ldots u_{k+1}^{\top}\right]^{\top}\right)=F_{i, j}\left(F_{i, j}^{k}\left(x,\left[u_{1}^{\top} \ldots u_{k}^{\top}\right]^{\top}\right), u_{k+1}\right) .
$$

$F_{i, j}^{k}$ has more control inputs compared to $F_{i, j}$ since a separate input value $u_{t}$ can be selected for each time step $1 \leq t \leq k$. Thus, $B \times Z_{u}$ is potentially a full dimensional set and a controller $u=-\kappa s$ can be found to pose a prescribed bound on the reachable set. Finding such a controller can be formulated again as a linear programming problem as explained in [2]. It is shown in [2] that such a feedback controller exists under a dynamics smoothness assumptions and bounded disturbance. A robustifiability measure can be computed that shows how well the error can be compensated with the feedback control. Robustifiability of the system $F_{i, j}$ at a state $s$ is defined as an inverse of the condition value of the matrix $\left[\frac{\partial F_{i, j}^{n}}{\partial u_{n}} \ldots \frac{\partial F_{i, j}^{n}}{\partial u_{1}}\right]$. The condition value can be obtained through singular value decomposition. In particular, for the vehicle model considered in this study, $\bar{F}_{i, j}^{3}$ can be shown to be robustifiable, meaning that a 3 -step combination of the MPs can keep the uncertainty bounded.

As a result, each MP becomes of the form $u^{f f}-\kappa e$, where $u^{f f}$ is a feed-forward term corresponding to the central value of the input cell $U_{j}$ (i.e. $Z_{u} . \mu$ ) and $-\kappa e$ is a feedback term applied to the error $e=x-Z_{x} . \mu$ between the current state and the center of the intended starting zonotope.

\section{Default dynamics}

To be able to move to different terrains with unknown dynamics, we will initially construct a default dynamical model $\bar{F}_{d}$. Let us label the terrain type where the rover starts exploration as $\mathcal{R}^{1}$. We probe the dynamics in this region and construct the locally linear dynamics $\bar{F}_{1, j}$ for each input cell $U_{j}: \bar{F}_{1, j}(x, u)=\{A x+B u+c\} \oplus \mathbf{W}$. We then add an additional terrain uncertainty disturbance $\mathbf{W}^{d}$ to the local dynamics to construct the default dynamics $\bar{F}_{d, j}(x, u)=\{A x+B u+c\} \oplus \mathbf{W} \oplus \mathbf{W}^{d}$. $\mathbf{W}^{d}$ is a design parameter that comes from an initial estimate on how different the dynamics may be on different terrains. A choice of small $\mathbf{W}^{d}$ may result in unsafe behavior, while an overly

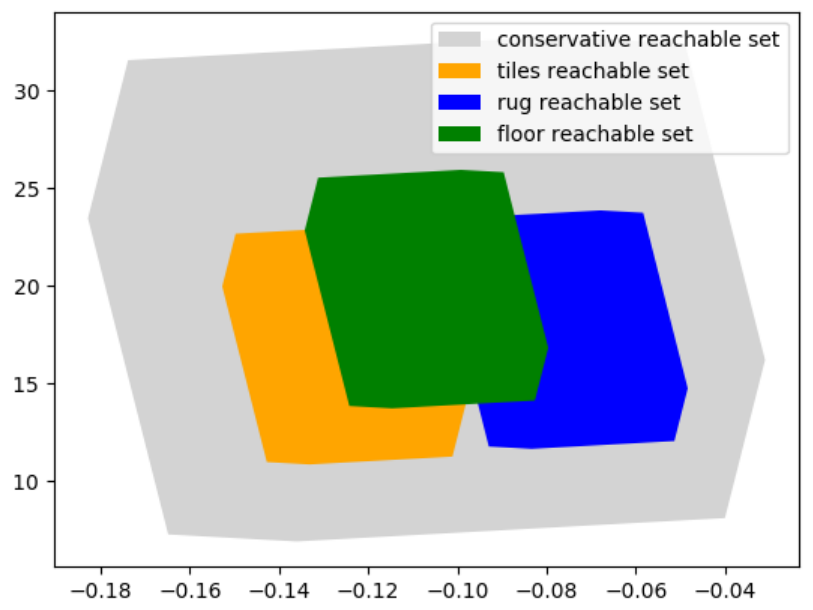

Fig. 3: Reachable sets for a left turning motion primitive on different terrain types.

large $\mathbf{W}^{d}$ will lead to very conservative primitives as every action will look unsafe.

In Fig. 3 we have demonstrated the reachable sets for a left turning motion primitive of a rover on different terrain types. The conservative reachable set corresponds to the default dynamics model that follows the floor dynamics model with an uncertainty estimate $\mathbf{W}^{d}$. The existence of a planning solution using a set of robust feedback motion primitives relies on the assumption that the robot will not lose mobility on any of the terrains, or formally: for each dimension there exists at least one motion primitive where the projection of the default model reachable set on that dimension is strictly positive or negative.

\section{EXPERIMENTS}

\section{A. Setup}

The approach was tested on an Erle Rover UGV in a room with slippery floor, partly covered by different materials representing different terrain types, namely a mat made of EVA foam tiles and a soft textured rug, see Fig. 4. Motion capture was used for localization of the rover. The motion planning and control synthesis were implemented in Python, using Robot Operating System (ROS) to interface with the rover and the motion capture equipment. The input space was two-dimensional, each input consisting of a steering angle and motor torque, and coarsely discretized with 6 MPs constant motion commands - corresponding to going straight, right and left in forward and backward drive respectively.

The exploration scenario was implemented as follows. The floor of the room, which constitutes the area to be explored, was represented as a rectangular grid consisting of $5 \times 5$ cells. Parts of the floor corresponding to certain grid cells were covered with mats or rugs, the texture of which was expected to have a different effect on the rover dynamics than that of a slippery floor. At the start of the exploration the rover was given a default dynamics model, which had been learned on the uncovered floor in advance. This model was 


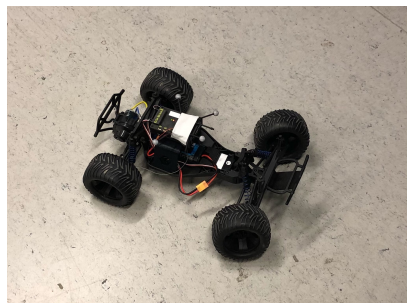

(a)

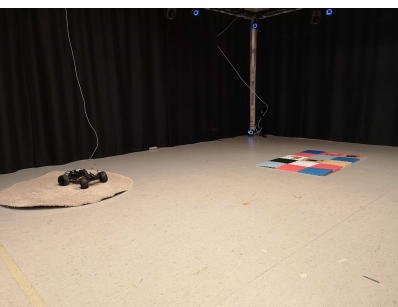

(b)
Fig. 4: Experimental setup. (a) Erle Rover. (b) Laboratory floor with two coverings representing different terrain types for dynamics identification.

subsequently used for motion planning and control during the entire exploration process.

Afterwards, to verify the utility of the information on the rover dynamics obtained during the exploration, several planning problems in a larger area consisting of regions with different known terrains were simulated. The learned models of the rover dynamics were incorporated into the planning algorithm to be used in the appropriate regions and the resulting plans were compared with the ones found by the default algorithm that did not account for differences in terrains.

\section{B. Terrain Dynamics Identification Results}

During the laboratory experiments, information on the rover dynamics on three types of terrain was collected. Fig. 5 shows an example of the data samples obtained by sending the same left, straight and right forward motion commands to the rover several times on each terrain. In the figure, each point represents a change in rover position in response to a single motion command. It can be clearly seen that, while there is a certain degree of variation in responses to the same command on a particular terrain, the responses on different terrains form identifiable clusters that signify differences in underlying rover dynamics.

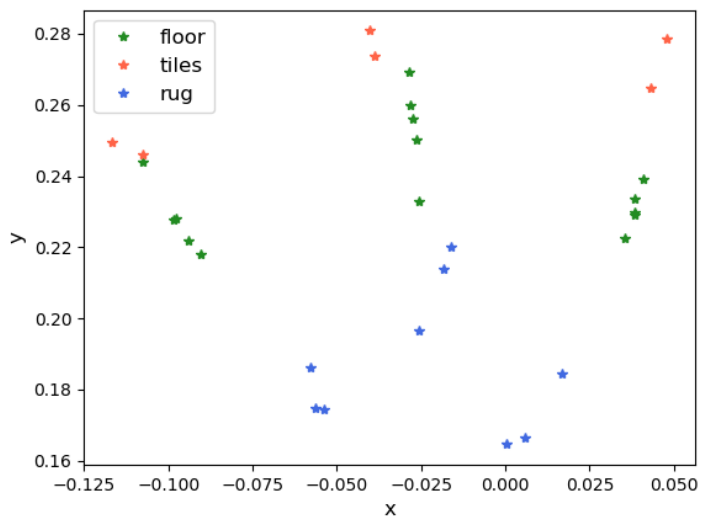

Fig. 5: Rover dynamics samples for the same left, straight and right forward motion commands on different terrains.

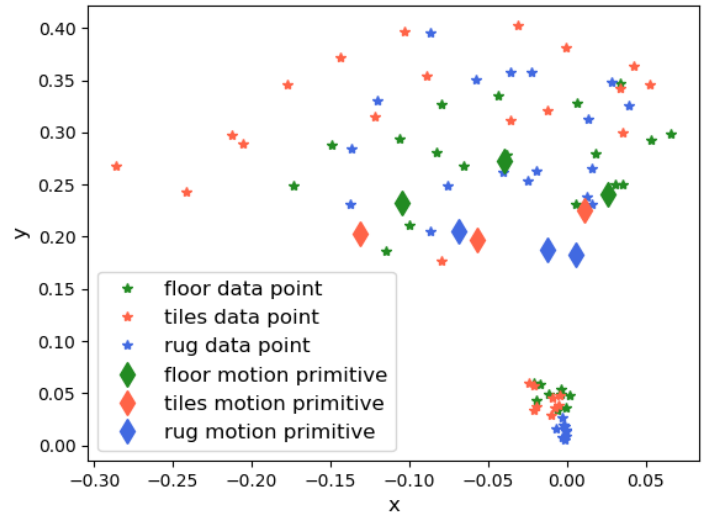

Fig. 6: Rover dynamics samples for different forward motion commands and the resulting approximations of motion primitive dynamics on different terrains.

The collected samples were used to construct models of the rover dynamics for each terrain. The resulting approximations of the dynamics for forward MPs together with the samples used in linear model fitting are shown in Fig. 6. In the figure, the points represent position changes in response to a single command received, with rhombuses representing the obtained approximations for the three MPs and stars corresponding to individual responses to different motion commands from the discretized input space.

Although the materials representing terrain types in the experiments were not drastically different, it is clear from Fig. 5 that the dynamics of the rover on them were distinct. It is especially obvious that the rug allowed less movement for the same motion commands than both the floor and the foam tiles, which is to be expected considering that the rug is moderately textured, while the two other surfaces are smooth. There is, however, a significant level of variance in the results of similar motion commands, which can be observed in Fig. 6. It can also be seen from the figure that this variance brings the MPs for different models closer to each other than one would have expected based on the previous figure.

\section{Motion Planning Results}

The models were then used in a series of planning problems in order to analyze the dynamics and the associated costs of traversing the same regions with the same aim but on different terrains. Fig. 7 provides examples of optimal paths found by $\mathrm{A}^{*}$ for the same problems assuming different terrains and, consequently, different rover dynamics. The problems depicted are: a simple diagonal traversal of a square area, a snake-like exploration of a grid and a $180^{\circ}$ orientation reversal in the same position. Table I summarizes the associated costs depending on the assumed terrain. The costs were computed as the total number of motion commands executed in the path as a proxy for how much time it takes to traverse the trajectory given that the MPs are fixed time. Alternatively, any cost function that can be evaluated for each MP, e.g., energy consumption, could be used. Computational costs of path planning are provided in Table II. 


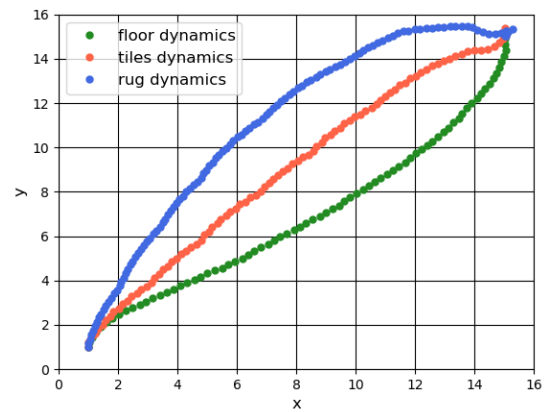

(a)

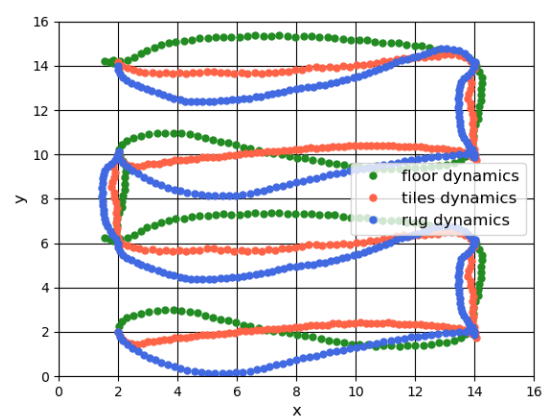

(b)

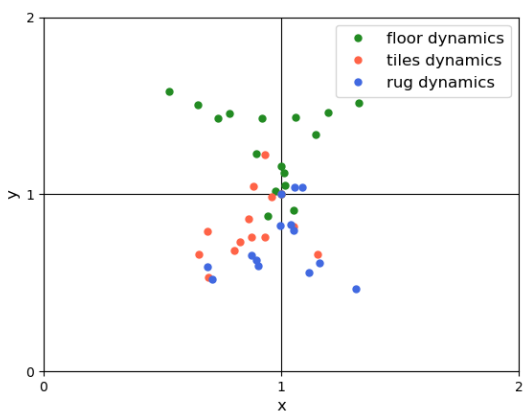

(c)

Fig. 7: Optimal paths obtained by A* with different dynamics models. (a) Diagonal traversal of a square area. (b) Grid exploration. (c) Orientation reversal.

To see the effect of incorporating the information about the rover dynamics on different terrains into the planning algorithm, three areas consisting of regions with different terrain types were simulated and a path through each area was planned first using only the default - floor - model and then using all three available models. The obtained paths can be seen in Fig. 8. In the figure, green represents the floor, pink - the tiles and blue - the rug terrain types.

The differences in the rover dynamics on different terrains are fairly obvious in the planned trajectories depicted in Fig. 7. For example, it can be seen that on the tiles the most efficient way to move was found to be almost entirely straight, while on the floor and on the rug more steering commands were used; this can be due to the higher range of turning on the tiles, as seen in Fig. 6. However, not
TABLE I: Costs of optimal paths on different terrains

\begin{tabular}{|c|c|c|c|}
\hline & Floor & Tiles & Rug \\
\hline Diagonal path & 78 & 91 & 114 \\
\hline Grid exploration path & 259 & 305 & 323 \\
\hline Orientation reversal path & 16 & 13 & 13 \\
\hline
\end{tabular}

TABLE II: Computational costs of path planning on different terrains

\begin{tabular}{|c|c|c|c|}
\hline & Floor & Tiles & Rug \\
\hline Diagonal path & $0.22 \mathrm{~s}$ & $1.17 \mathrm{~s}$ & $2.14 \mathrm{~s}$ \\
\hline Grid exploration path & $6.08 \mathrm{~s}$ & $1.16 \mathrm{~s}$ & $1.27 \mathrm{~s}$ \\
\hline Orientation reversal path & $1.75 \mathrm{~s}$ & $0.71 \mathrm{~s}$ & $0.12 \mathrm{~s}$ \\
\hline
\end{tabular}

much can be concluded about the terrain traversability by simply studying the trajectories. The costs of each trajectory provided in Table I offer much more insight in that respect. It is clear from the values in the table that the floor is preferable to both the tiles and the rug for efficient traversal, with the rug being the most expensive terrain to traverse. This is, once again, consistent with the intuition that the rug's texture would provide some additional resistance due to friction. The floor, meanwhile, is slippery and therefore the friction is minimal. It is notable that both the tiles and the rug models slightly outperformed the floor one in the scenario of orientation reversal without changing the position. A possible explanation for that would be that, since the rover generally moves farther after one motion command on the floor, it would need more steps to return to the original position. The path trajectory depicted in Fig. 7c seems to support this explanation.

The results of incorporating all the dynamics models into the planning algorithm are also consistent with the conclusion that the floor allows the most efficient traversal. In Fig. 8a it can be seen that, without taking different terrains and their dynamics into account, the planned trajectory goes through both the pink region (representing the tiles) and the blue region (representing the rug). Meanwhile, the trajectory in Fig. 8b clearly aims to avoid both, even if not fully. Similar behavior, if less pronounced, can be observed in the more complex environments depicted in Fig. 8c-8f. As discussed above, both the pink and the blue regions are more expensive to traverse and, therefore, avoiding them means avoiding the extra costs associated with the terrain-specific dynamics. However, since the terrains in this case are not substantially different, avoiding either region entirely would require a path through the cheapest terrain long enough that its overall cost would still be higher than that of a shorter path that goes partially through the more expensive terrains. One can expect that the less movement a terrain allows, the more weight the need to avoid it would have, even if the only way to achieve this is by making the path significantly longer.

\section{CONCLUSIONS AND FUTURE WORK}

In this paper we proposed a data-driven approach to construct terrain-specific MPs for a UGV by probing the dynamics on each terrain. We further showed that terrain- 


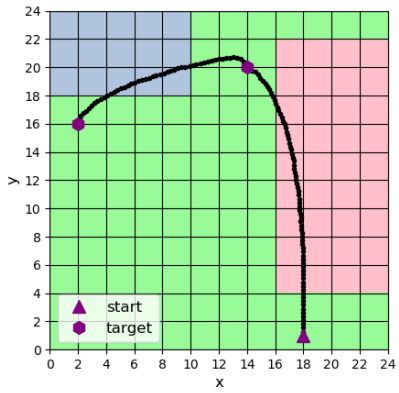

(a)

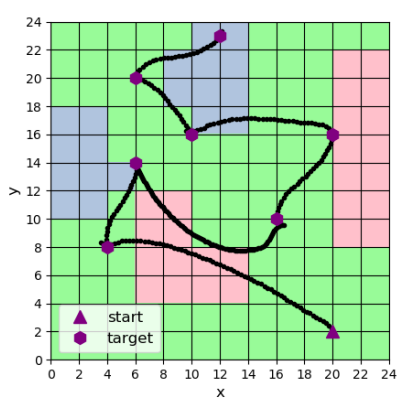

(c)

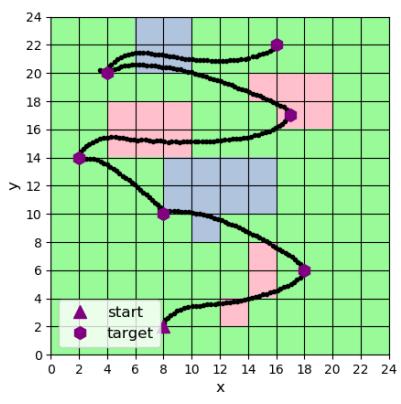

(e)

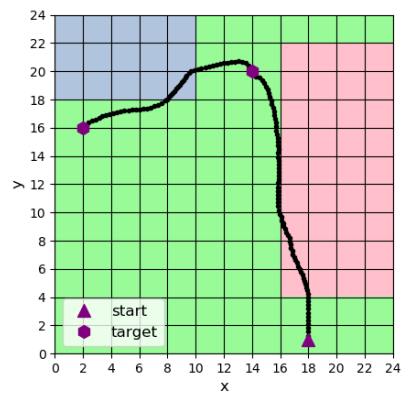

(b)

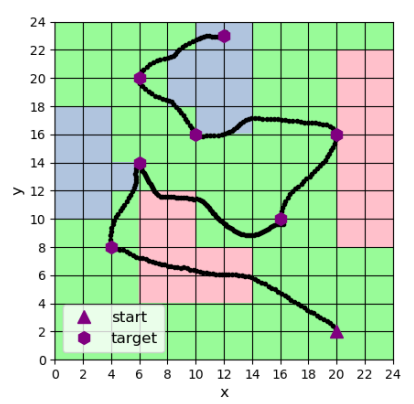

(d)

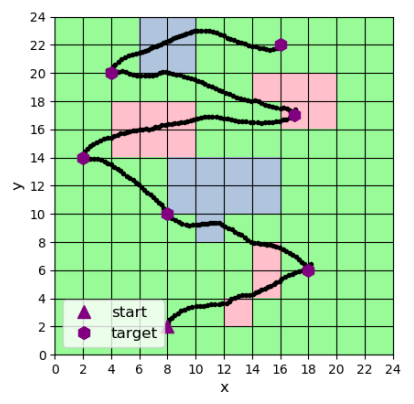

(f)
Fig. 8: Optimal paths obtained by $\mathrm{A}^{*}$ in a multi-terrain area. (a), (c), (e) Paths obtained without taking different terrain dynamics into account. (b), (d), (f) Paths obtained using all the available information about terrain dynamics.

specific MPs are beneficial for path optimization in motion planning problems in multi-terrain environments.

A key assumption in this work is that while the dynamics on each terrain type is not known, their borders are known so that probing can be carried out inside each terrain region. In addition, the dynamics identification of the terrains is an independent task requiring pro-active probing of each terrain. We aim to address these two aspects in our future work by investigating how detected changes in the dynamics for one of the MPs can be transferred to others; this will allow to identify terrain dynamics without explicit trial of every MP, potentially enabling the use of a wider range of MPs.

\section{REFERENCES}

[1] J. A. Starek, B. Açıkmeşe, I. A. Nesnas, and M. Pavone, "Spacecraft autonomy challenges for next-generation space missions," in Advances in Control System Technology for Aerospace Applications. Springer, 2016, pp. 1-48.

[2] P. Tajvar, A. Varava, D. Kragic, and J. Tumova, "Robust motion planning for non-holonomic robots with planar geometric constraints," in The International Symposium on Robotics Research October 6-10, 2019, Hanoi, Vietnam, 2019.

[3] P. Polack, F. Altché, B. d'Andréa Novel, and A. de La Fortelle, "The kinematic bicycle model: A consistent model for planning feasible trajectories for autonomous vehicles?" in 2017 IEEE Intelligent Vehicles Symposium (IV). IEEE, 2017, pp. 812-818.

[4] A. Majumdar and R. Tedrake, "Funnel libraries for real-time robust feedback motion planning," The International Journal of Robotics Research, vol. 36, no. 8, pp. 947-982, 2017.

[5] M. Vukosavljev, Z. Kroeze, M. E. Broucke, and A. P. Schoellig, "A framework for multi-vehicle navigation using feedback-based motion primitives," in 2017 IEEE/RSJ International Conference on Intelligent Robots and Systems (IROS). IEEE, 2017, pp. 223-229.

[6] A. Hait, T. Siméon, and M. Taïx, "Robust motion planning for rough terrain navigation," in IN IEEE INT. CONF. ON INTELLIGENT ROBOTS AND SYSTEMS KYONGJU, KOREA. Citeseer, 1999.

[7] T. Ersson and X. Hu, "Path planning and navigation of mobile robots in unknown environments," in Proceedings 2001 IEEE/RSJ International Conference on Intelligent Robots and Systems. Expanding the Societal Role of Robotics in the the Next Millennium (Cat. No. 01CH37180), vol. 2. IEEE, 2001, pp. 858-864.

[8] N. E. Du Toit and J. W. Burdick, "Robot motion planning in dynamic, uncertain environments," IEEE Transactions on Robotics, vol. 28 , no. 1, pp. 101-115, 2011.

[9] M. Bujarbaruah, X. Zhang, M. Tanaskovic, and F. Borrelli, "Adaptive MPC under time varying uncertainty: Robust and stochastic," arXiv preprint arXiv:1909.13473, 2019.

[10] N. Wagener, C.-A. Cheng, J. Sacks, and B. Boots, "An online learning approach to model predictive control," arXiv preprint arXiv: 1902.08967, 2019.

[11] X. Zhang, M. Bujarbaruah, and F. Borrelli, "Near-optimal rapid MPC using neural networks: A primal-dual policy learning framework," IEEE Transactions on Control Systems Technology, 2020.

[12] R. McAllister, T. Peynot, R. Fitch, and S. Sukkarieh, "Motion planning and stochastic control with experimental validation on a planetary rover," in 2012 IEEE/RSJ International Conference on Intelligent Robots and Systems. IEEE, 2012, pp. 4716-4723.

[13] M. Rafieisakhaei, A. Tamjidi, S. Chakravorty, and P. R. Kumar, "Feedback motion planning under non-gaussian uncertainty and nonconvex state constraints," in 2016 IEEE International Conference on Robotics and Automation (ICRA). IEEE, 2016, pp. 4238-4244.

[14] W. Han and R. Tedrake, "Local trajectory stabilization for dexterous manipulation via piecewise affine approximations," in 2020 IEEE International Conference on Robotics and Automation (ICRA). IEEE, 2020, pp. 8884-8891.

[15] P. Papadakis, "Terrain traversability analysis methods for unmanned ground vehicles: A survey," Engineering Applications of Artificial Intelligence, vol. 26, no. 4, pp. 1373-1385, 2013.

[16] E. Coyle, E. G. Collins, and L. Lu, "Updating control modes based on terrain classification," in 2010 IEEE International Conference on Robotics and Automation. IEEE, 2010, pp. 4417-4423.

[17] L. Lu, C. Ordonez, E. G. Collins Jr, E. Coyle, and D. Palejiya, "Terrain surface classification with a control mode update rule using a $2 \mathrm{~d}$ laser stripe-based structured light sensor," Robotics and Autonomous Systems, vol. 59, no. 11, pp. 954-965, 2011.

[18] A. Howard and H. Seraji, "Vision-based terrain characterization and traversability assessment," Journal of Robotic Systems, vol. 18, no. 10, pp. 577-587, 2001.

[19] D. Belter, P. Łabkecki, and P. Skrzypczyński, "Adaptive motion planning for autonomous rough terrain traversal with a walking robot," Journal of Field Robotics, vol. 33, no. 3, pp. 337-370, 2016.

[20] P. Krüsi, P. Furgale, M. Bosse, and R. Siegwart, "Driving on point clouds: Motion planning, trajectory optimization, and terrain assessment in generic nonplanar environments," Journal of Field Robotics, vol. 34, no. 5, pp. 940-984, 2017.

[21] H. J. Van Waarde, J. Eising, H. L. Trentelman, and M. K. Camlibel, "Data informativity: a new perspective on data-driven analysis and control," IEEE Transactions on Automatic Control, vol. 65, no. 11, pp. 4753-4768, 2020. 